\title{
Insulin resistance and low urinary citrate excretion in calcium stone formers
}

\author{
A. Cupisti ${ }^{\text {a,* }}$, M. Meola ${ }^{\text {a }}$, C. D’Alessandro ${ }^{\text {a }, ~ G . ~ B e r n a b i n i ~}{ }^{\text {a }}$, \\ E. Pasquali ${ }^{\text {a }}$, A. Carpi ${ }^{\text {b }}$, G. Barsotti ${ }^{\text {a }}$ \\ a Department of Internal Medicine, Nephrology Unit, University of Pisa, Via Roma 67, 56126 Pisa, Italy \\ ${ }^{\mathrm{b}}$ Department of Reproduction and Ageing, Nephrology Unit, University of Pisa, Pisa, Italy
}

Received 16 March 2006; accepted 27 September 2006

Available online 4 December 2006

\begin{abstract}
Epidemiological data suggest an association between kidney stones and some features of metabolic syndrome such as an overweight condition, arterial hypertension or glucose intolerance. However, mechanisms remain to be elucidated. This study aimed to evaluate insulin resistance, as assessed by homeostasis model assessment (HOMA-IR), and urine composition analysis in patients affected by calcium nephrolithiasis. A cohort of 61 (38 male, 29-57 years of age) non-diabetic calcium stone formers was studied. Data about body mass index, arterial blood pressure, serum biochemistry including parathyroid hormone and calcitriol were recorded in all the patients; fasting glucose and insulin were determined to calculate HOMA-IR value and accordingly the patients were grouped into tertiles. Urine $\mathrm{pH}$ and urinary excretion of calcium, citrate, phosphate, oxalate, uric acid, urea and creatinine were measured on $24 \mathrm{~h}$ urine samples. Patients of the highest HOMA-IR tertile showed lower urine citrate levels than patients of the lowest HOMA-IR tertile (475 $\pm 243 \mathrm{vs}$. $630 \pm 187 \mathrm{mg} / 24 \mathrm{~h}, p<0.05)$, whereas no difference was detected as far as urinary oxalate, calcium, uric acid, phosphate, and urine $\mathrm{pH}$ and urine volume output were concerned. HOMA-IR values were positively related to uric acid serum levels $(r=0.31, p<0.05)$ and negatively to urinary citrate excretion $(r=-0.26, p<0.05)$. Hypocitraturic patients showed higher levels of HOMA-IR than normocitraturic ones $(3.03 \pm 0.92$ vs. $2.25 \pm 1.19, p<0.05)$. This study shows that a higher level of insulin resistance is associated with lower urinary citrate excretion, and that hypocitraturic patients show a greater insulin resistance than normocitraturic calcium stone formers. This may be related to changes in citrate, $\mathrm{Na}^{+}-\mathrm{K}^{+}$and $\mathrm{H}^{+}$renal tubule transports, which have been described in insulin resistance. In conclusion, insulin resistance may contribute to an increased risk of calcium stone formation by lowering urinary citrate excretion. This finding suggests the need for a careful metabolic assessment in patients known to form calcium stones in order to ensure stone recurrence prevention and cardiovascular protection.
\end{abstract}

(C) 2006 Elsevier Masson SAS. All rights reserved.

Keywords: Insulin resistance; Kidney stone; Urinary citrate; Urinary calcium; Hypocitraturia

\section{Introduction}

Calcium nephrolithiasis is a quite frequent disease in developed countries. Approximately $10 \%$ of men and $5 \%$ of women will experience a symptomatic kidney stone by the age of 75 years and about $80 \%$ of kidney stones contain calcium [1-3]. The identification of risk factors for nephrolithiasis

\footnotetext{
* Corresponding author. Tel.: +39050 993405; fax: +39050993110.

E-mail address: acupisti@med.unipi.it (A. Cupisti).
}

may be helpful in the primary and secondary prevention of kidney stone formation [4-6]. Epidemiological studies report an association between nephrolithiasis and diabetes, obesity, overweight [7-9] or arterial hypertension [10,11], but most of the studies did not give information about the stone composition. However, the majority of patients were presumably affected by calcium stone disease, since it is largely the most frequent type in nephrolithiasis adult patients. In Western countries, a growing population is affected by obesity and/or metabolic syndrome, a condition defined by the clustering of abdominal obesity, dyslipidaemia, elevated blood pressure, 
and elevated fasting plasma glucose [12,13]. This emerging condition is associated also with hyperuricaemia, coronary artery disease and sympathetic overactivity, resulting in a higher rate of cardiovascular events [12,13]. The metabolic syndrome is considered a condition of insulin resistance.

When the different types of stone composition are taken into account, evidence exists that insulin resistance is associated with uric acid stone disease [14]. This is in keeping with some reports showing that uric stone formers are more frequently hypertensive and overweight, while exhibiting higher plasma levels of both triglycerides and uric acid $[15,16]$.

The association between insulin resistance and uric acid stone disease [14] can be explained by a defect in urine acidification, namely to an impaired L-glutamine system and reduction of $\mathrm{Na}^{+}-\mathrm{K}^{+}$transport at the proximal tubule. This change results in very low urine $\mathrm{pH}$ which is the main risk factor for uric acid stone formation. A defect of the $\mathrm{Na}^{+}-\mathrm{K}^{+}$and $\mathrm{H}^{+}$transport systems could also be associated with lower citrate excretion, which is a well known risk factor for calcium stone formation [4-6]. In addition, insulin may also affect renal handling of calcium and phosphate, which are factors implicated in calcium stone formation as well [17-19]. This study aimed to evaluate the association between risk factors for calcium stone formation and insulin resistance in a cohort of non-diabetic calcium stone formers.

\section{Patients and methods}

Sixty-one non-diabetic calcium stone formers (38 males, 23 females, $29-57$ years of age) were included in the study. Diagnosis was proven by chemical analysis of passed stones or by the detection of Rx-opaque kidney stones. Patients with uric acid, struvite or cystine stones or with urinary tract infection were excluded. Renal tubular acidosis, primary hyperparathyroidism or sarcoidosis were considered as exclusion criteria, as well as chronic liver disease, renal insufficiency or chronic pancreatitis. No patient was on steroid or immunosuppressive therapy. At the time of the study, the patients were studied without any drugs able to interfere with calcium or citrate excretion, namely no patient assumed diuretics or potassium citrate. Some hypertensive patients were on therapy with calcium-channel blockers (5 cases), ACE-inhibitors or angiotensin II receptor blockers (6 cases), or beta-blockers (3 cases).

We have not systematically performed dietary records in all the patients. However, our cohort is quite homogeneous for race and lifestyle, presuming that the majority followed a Mediterranean diet, with a mean sodium intake of $197 \pm$ $97 \mathrm{mmol} / 24 \mathrm{~h}$.

The history of the patients selected for the study showed a single stone episode in 21 of them. In the patients with recurrences, the rate was 0.39 stones/patient per year, before our observation.

Blood samples were taken after an overnight fasting to measure serum levels of creatinine, urea, uric acid, calcitriol, parathyroid hormone (PTH), glucose, calcium, phosphate, alkaline phosphates, magnesium, sodium, potassium, total cholesterol, high-density lipoprotein (HDL) cholesterol, and triglycerides. Fasting levels of insulin were determined using an immunoradiometric assay (DiaSorin, Italy). Insulin resistance was assessed using the validated homeostasis model assessment (HOMA-IR) [20], based on fasting immunoreactive insulin and glucose plasma levels. It was calculated by the following formula: HOMA-IR = fasting immunoreactive insulin $(\mu \mathrm{U} / \mathrm{ml}) *$ fasting glucose $(\mathrm{mmol} / \mathrm{l}) / 22.5$. It is assumed that low HOMA-IR values mean normal insulin sensitivity whereas high values mean insulin resistance. According to tertiles of HOMA-IR values, the studied population was divided into three groups with low, middle and high HOMA-IR values.

Samples from $24 \mathrm{~h}$ urine collections, using thymol as preservative, were used to measure daily urinary excretion of calcium, citrate, phosphate, oxalate, urea, creatinine and $\mathrm{pH}$. We defined hypercalciuria as a daily urinary calcium excretion $>4 \mathrm{mg} / \mathrm{kg}$ body weight, hyperoxaluria as a urinary oxalate $>42 \mathrm{mg} /$ day, hyperuricuria as an uric acid excretion $>700 \mathrm{mg}$ in males and $600 \mathrm{mg}$ in females. Hypocitraturia was defined as an urinary citrate excretion of $<320 \mathrm{mg} /$ day.

Urinary oxalate was determined by an enzymatic method using oxalate oxidase, and urinary citrate was assayed by the citrate lyase method, after sample centrifugation for separation of insoluble substances. All the other determinations were assessed using the routine laboratory methods.

Dietary protein intake was calculated using the urea appearance method according to Maroni's formula [21], and expressed as protein catabolic rate normalized by $\mathrm{kg}$ of body weight (nPCR).

\subsection{Statistical analysis}

Descriptive statistics are expressed as mean \pm standard deviation. Statistical evaluation was performed using a statistical package (Excel for Windows) for personal computer. Differences between groups were evaluated by the Student's $t$-test for unpaired data, and the one-way analysis of variance (one-way ANOVA). Pearson's correlation test and multivariate regression analysis were performed. Statistical differences were considered significant when $p<0.05$.

\section{Results}

In the studied population, the prevalence of the main metabolic risk factors of calcium stone disease resulted the following: $28 \%$ hypercalciuria, $25 \%$ hypocitraturia, $20 \%$ hyperuricosuria and $11 \%$ hyperoxaluria. In the same patient, hypercalciuria was associated with hypocitraturia in 3 cases, with hyperuricosuria in 6 cases and with hyperoxaluria in 2 cases. No patient showed more than two of these conditions associated.

Eleven percent of patients were overweight, and $9 \%$ showed a BMI $>30 \mathrm{~kg} / \mathrm{m}^{2}$.

Patients in the highest HOMA-IR tertiles showed lower citrate levels than patients in the middle or the lowest tertile 
Table 1

Clinical and biochemical characteristics of the patients of the highest HOMAIR tertile and of the lowest HOMA-IR tertile

\begin{tabular}{lccl}
\hline & High HOMA-IR & Low HOMA-IR & $p$ \\
\hline Age, years & $40.8 \pm 11.9$ & $42.6 \pm 13.6$ & n.s. \\
Body weight, kg & $74.4 \pm 16.6$ & $73.5 \pm 19.5$ & n.s. \\
BMI, kg/m & $25.8 \pm 4.6$ & $24.7 \pm 4.7$ & n.s. \\
nPCR, g/kg/body wt & $1.1 \pm 0.2$ & $1.1 \pm 0.2$ & n.s. \\
Systolic BP, mmHg & $131.2 \pm 18.6$ & $126.6 \pm 15.9$ & n.s. \\
Diastolic BP, mmHg & $82.0 \pm 9.3$ & $81.5 \pm 7.4$ & n.s. \\
& & & \\
Serum & & & \\
Potassium, mEq/l & $3.8 \pm 0.3$ & $3.9 \pm 0.3$ & n.s. \\
Calcium, mg/dl & $9.4 \pm 0.5$ & $9.4 \pm 0.3$ & n.s. \\
Phosphate, mg/dl & $3.3 \pm 0.6$ & $3.1 \pm 0.7$ & n.s. \\
Uric acid, mg/dl & $6.3 \pm 1.3$ & $5.2 \pm 1.1$ & $<0.01$ \\
PTH, pg/ml & $31.8 \pm 18.1$ & $35.7 \pm 18.8$ & n.s. \\
1,25-Vitamin D, pg/ml & $50.5 \pm 21.4$ & $53.1 \pm 18.3$ & n.s. \\
Insulin, mU/ml & $16.0 \pm 3.4$ & $6.3 \pm 1.6$ & $<0.001$ \\
Glucose, mg/dl & $96 \pm 13$ & $87 \pm 7$ & $<0.01$ \\
Triglycerides, mg/dl & $136 \pm 64$ & $129 \pm 75$ & n.s. \\
HDL cholesterol, mg/dl & $50 \pm 10$ & $59 \pm 12$ & $<0.05$ \\
Total cholesterol, mg/dl & $221 \pm 39$ & $212 \pm 34$ & n.s. \\
\hline
\end{tabular}

HOMA-IR, insulin resistance as assessed by homeostasis model assessment; BMI, body mass index; nPCR, normalized protein catabolic rate; $\mathrm{BP}$, blood pressure; PTH, parathyroid hormone; HDL, high-density lipoprotein; n.s., not significant.

(Table 1). No difference at all was detected among the HOMA-IR groups as far as urinary oxalate, calcium, uric acid and phosphate were concerned. As expected, the highest HOMA-IR tertile showed higher fasting serum levels of insulin and uric acid. Accordingly, correlation analysis demonstrated that HOMA-IR values were positively related to uric acid serum levels $(r=0.31, p<0.05)$ and negatively to urinary citrate excretion $(r=-0.26, p<0.05)$ (Fig. 1). No statistically significant correlation resulted between HOMA-IR and the other urinary parameters studied.

Patients with hypocitraturia showed higher levels of HOMA-IR values than patients with normal values $(3.03 \pm 0.92$ vs. $2.25 \pm 1.19, p<0.05)$; nine out of the 20

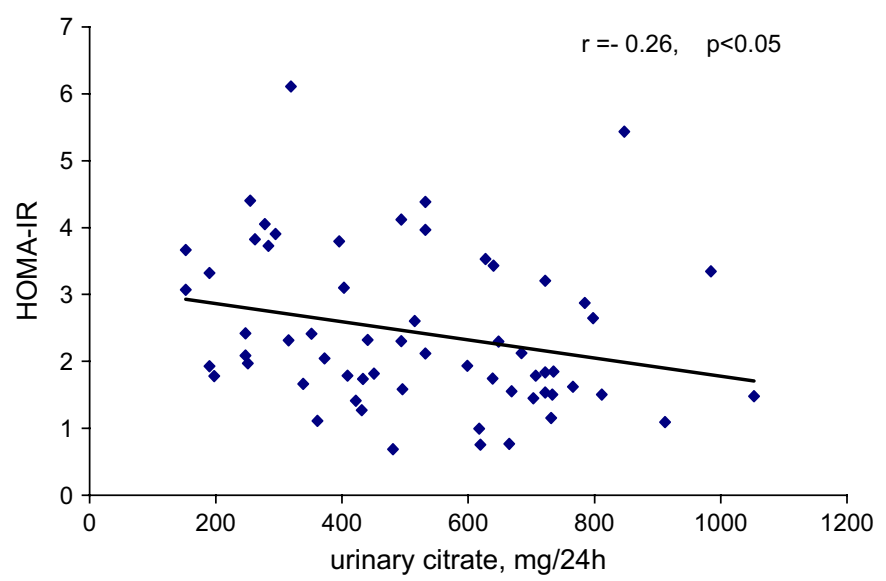

Fig. 1. Relationship between urinary citrate excretion and insulin resistance, as assessed by homeostasis model assessment (HOMA-IR), in the 61 patients with calcium stone disease.
Table 2

Renal function and urine composition characteristics of the patients of the highest HOMA-IR tertile and of the lowest HOMA-IR tertile

\begin{tabular}{lccl}
\hline & High HOMA-IR & Low HOMA-IR & $p$ \\
\hline Urine & & & \\
Volume output, ml/24 h & $1839 \pm 634$ & $2065 \pm 573$ & n.s. \\
$\mathrm{pH}$ & $5.7 \pm 0.4$ & $5.7 \pm 0.5$ & n.s. \\
Calcium, $\mathrm{mg} / 24 \mathrm{~h}$ & $231 \pm 115$ & $264 \pm 129$ & n.s. \\
Phosphate, $\mathrm{mg} / 24 \mathrm{~h}$ & $815 \pm 344$ & $792 \pm 213$ & n.s. \\
Oxalate, $\mathrm{mg} / 24 \mathrm{~h}$ & $24.6 \pm 7.1$ & $25.9 \pm 4.4$ & n.s. \\
Citrate, $\mathrm{mg} / 24 \mathrm{~h}$ & $475 \pm 243$ & $630 \pm 187$ & $<0.05$ \\
Uric acid, $\mathrm{mg} / 24 \mathrm{~h}$ & $565 \pm 258$ & $555 \pm 200$ & n.s. \\
Creatinine, $\mathrm{mg} / 24 \mathrm{~h}$ & $1594 \pm 508$ & $1585 \pm 379$ & n.s. \\
& & & \\
Creatinine clearance, $\mathrm{ml} / \mathrm{min}$ & $119 \pm 33$ & $113 \pm 27$ & n.s. \\
\hline
\end{tabular}

patients of the highest HOMA-IR tertile were hypocitraturic versus no patients from the lowest tertile (Table 2).

\section{Discussion}

Epidemiologic studies show that the incidence of nephrolithiasis increases in patients exhibiting an overweight condition, hypertension, dyslipidaemia and glucose intolerance. In patients with uric acid stone disease, evidence exists that the relationship between kidney stone formation and insulin resistance is represented by reduced proximal tubule ammoniagenesis and marked decrease of urine $\mathrm{pH}$. However, up to now few data exist in the literature about the relationship between urine composition and insulin resistance in calcium-stone formers.

The results of our study show that higher insulin resistance is associated with lower urinary citrate excretion, and that hypocitraturic patients show a higher insulin resistance than normocitraturic patients. Instead, protein intake, body mass index, renal function, serum PTH, calcitriol, and the urinary excretion of calcium, uric acid, oxalate and phosphate were quite similar among the three groups of patients with different HOMA-IR values. Our data suggest that insulin resistance may contribute to calcium stone formation by reducing urinary citrate excretion. The lower citrate excretion in the presence of insulin resistance may be caused by a defect in renal ammonium production or by changes in $\mathrm{Na}^{+}-\mathrm{K}^{+}$and $\mathrm{H}^{+}$transport mechanisms of the renal tubules $[8,14]$. Patients with insulin resistance have high levels of plasma free fatty acids, which can enter the proximal tubule cells and interfere with the utilization of glutamine; since free fatty acids constitute an alternative metabolic substrate for the proximal tubule cells, it results a reduction in glutamine usage and ammoniagenesis [22-24]. In addition, insulin resistance may directly impair ammoniagenesis as demonstrated by in vitro studies which showed that insulin is able to stimulate renal ammonium production from L-glutamine $[25,26]$. Insulin may also play a role in the activity of the proximal renal tubule $\mathrm{Na} / \mathrm{K}$ exchanger involved in transport or ionic trapping of ammonium in the tubular lumen [27]. Thus patients with insulin resistance may have an impaired ability to excrete ammonia so leading to hyperacidic urine [14], the main risk factor for uric acid stone formation 
$[28,29]$. Moreover, this condition can affect renal handling of citrate, i.e. it can increase apical membrane citrate uptake as long as citrate metabolism is highly maintained, leading to hypocitraturia, which is a major risk factor for calcium stone formation [4-6].

We have not observed any difference in calcium excretion. However, evidence exists that the compensatory hyperinsulinaemia of insulin resistance may increase urinary calcium excretion $[17,18]$ by decreasing the tubular reabsorption of filtered calcium [28], favouring urinary oversaturation for calcium phosphate and calcium oxalate.

In summary, our data show that a greater level of insulin resistance is associated with lower urinary citrate excretion, and that hypocitraturic patients show more insulin resistance than normocitraturic patients. This may be related to changes in citrate, $\mathrm{Na}^{+}-\mathrm{K}^{+}$and $\mathrm{H}^{+}$transport of the renal tubule, which has been described in conditions of insulin resistance. A larger cohort of patients and more sensitive methods to assess insulin resistance will be needed to confirm the association we observed between insulin resistance and low urine citrate in calcium stone patients.

It can be speculated that insulin resistance is more likely associated with uric stone formation when defective urinary acidification results in changes in urine $\mathrm{pH}$, namely hyperacidic urine, whereas it is more likely associated with calcium stone formation when a decrease of urinary citrate excretion occurs without significant reduction of urine $\mathrm{pH}$.

In the present study, the insulin resistance was estimated by HOMA-IR and not by euglycaemic hyperinsulinaemic clamp: this may be considered as a limitation of the study. However, a fairly good correlation exists between HOMA-IR and clampderived measures in adults [30], pregnancy [31] and renal dysfunction [32], suggesting that HOMA-IR may be a quite simple and useful tool for the screening of insulin resistance in a clinical setting and in population studies.

In conclusion, this study shows an association between the HOMA-IR values and changes in urinary citrate excretion in calcium stone formers. It can be speculated that insulin resistance may contribute, in part at least, to lowering urinary citrate excretion and thus to an increased risk of stone formation. This finding suggests the need for a careful metabolic assessment in patients known to form kidney stones in order to ensure effective stone recurrence prevention and cardiovascular protection.

\section{References}

[1] Stamatelou KK, Francis ME, Jones CA, Nyberg LM, Curhan GC. Time trends in reported prevalence of kidney stones in the United States: 1976-1994. Kidney Int 2003;63:1817-23.

[2] Johnson CM, Wilson DM, O'Fallon WM, Malek RS, Kurland LT. Renal stone epidemiology: A 25-year study in Rochester, Minnesota. Kidney Int 1979;16:624-31.

[3] Hiatt RA, Dales LG, Friedman GD, Hunkeler EM. Frequency of urolithiasis in a prepaid medical care program. Am J Epidemiol 1982;115: 255-65.

[4] Coe FL, Parks JH, Asplin JR. The pathogenesis and treatment of kidney stones. N Engl J Med 1992;327:1141-52.
[5] Levy FL, Adams-Huet B, Pak CY. Ambulatory evaluation of nephrolithiasis: an update of 1980 protocol. Am J Med 1995;98:50-9.

[6] Cupisti A, Morelli E, Lupetti S, Meola M, Barsotti G. Low urine citrate excretion as main risk factor for recurrent calcium oxalate nephrolithiasis in males. Nephron 1992;61:73-6.

[7] Taylor EN, Stampfer MJ, Curhan GC. Obesity, Weight Gain, and the risk of Kidney stones. JAMA 2005;293:455-62.

[8] Taylor EN, Stamper MJ, Curhan GC. Diabetes mellitus and the risk of nephrolithiasis. Kidney Int 2005;68:1230-5.

[9] Pak CY, Sakhaee K, Moe OW, Preminger GM, Poindexter JR, Peterson RD, et al. Biochemical profile of stones forming patients with diabetes mellitus. Urology 2003;61:523-7.

[10] Cappuccio FP, Strazzullo P, Mancini M. Kidney stones and hypertension: Population-based study of an independent clinical association. Br J Med 1990;300:1234-6.

[11] Borghi L, Meschi T, Guerra A, Briganti A, Schianchi T, Allegri F, et al. Essential arterial hypertension and stone disease. Kidney Int 1999; 55:2397-406.

[12] Kahn R, Buse J, Ferranini E, Stern M. The metabolic syndrome: time for a critical appraisal. Diabetologia 2005;48:1684-99.

[13] Cohn GS, Kittleson MM, Blumenthal RS. Toward an improved diagnosis of the metabolic syndrome: Other clues to the presence of insulin resistance. Am J Hypertens 2005;18:1099-103.

[14] Abate N, Chandalia M, Cabo-Chan Jr AV, Moe OW, Sakhaee K. The metabolic syndrome and uric acid nephrolithiasis: Novel features of renal manifestation of insulin resistance. Kidney Int 2004;65:386-92.

[15] Cupisti A, Setti G, Morelli E, Meola M, Lenti C, Paparatto P, et al. Calcolosi di acido urico ed ipertensione arteriosa. Giorn Ital Nefrol 1997; 14:37-41

[16] Daudon M, Lacour B, Jungers P. Influence of body size on urinary stone composition in men and women. Urol Res 2006;34:193-9.

[17] Kerstetter J, Caballero B, O’Brien K, Wurtman R, Allen L. Mineral homeostasis in obesity: Effects of euglycemic hyperinsulinemia. Metabolism 1991;40:707-13.

[18] Shimamoto K, Higashiura K, Nakagawa M, Masuda A, Shiiki M, Miyazaki Y, et al. Effects of hyperinsulinemia under the euglycemic condition in calcium and phosphate metabolism in non-obese normotensive subjects. Tohoku J Exp Med 1995;177:271-8.

[19] Schwille PO, Schmiedt A, Herrmann U. Post prandial hyperinsulinaemia, insulin resistance and inappropriately high phosphaturia are features of younger males with idiopathic calcium urolithiasis: attenuation by ascorbic acid supplementation of a test meal. Urol Res 1997;25:45-9.

[20] Matthews DR, Hosker JP, Rudenski AS, Naylor BA, Treacher DF, Turner RC, et al. Homeostasis model assessment: Insulin resistance and beta-cell function from fasting plasma glucose and insulin concentration in man. Diabetologia 1985;28:412-9.

[21] Maroni BJ, Steinman TI, Mitch WE. A method for estimating nitrogen intake in patients with chronic renal failure. Kidney Int 1985;27: $58-65$.

[22] Bagnasco SM, Gaydos DS, Risques A, Preuss HG. The regulation of renal ammoniagenesis in the rat by extracellular factors. III. Effects of various fuels on in vitro ammoniagenesis. Metabolism 1983;32:900-5.

[23] Lemieux G, Vinay P, Gougoux A, Baverel G, Cartier P. Relationship between the renal metabolism of glutamine, fatty acids and ketone bodies. Curr Probl Clin Biochem 1977;8:379-88.

[24] Vinay P, Lemieux G, Cartier P, Ahmad M. Effects of fatty acids on renal ammoniagenesis in vivo and in vitro studies. Am J Physiol 1976;231: $880-7$.

[25] Chobanian MC, Hammerman MR. Insulin stimulates ammoniagenesis in canine renal proximal tubular segments. Am J Physiol 1987;253: F1171-7.

[26] Krivosikova Z, Spustova V, Dzurik R. Participation of P-dependent and P-independent glutaminases in rat kidney ammoniagenesis and their modulation by metabolic acidosis, hippurate and insulin. Physiol Res 1998;47:177-83.

[27] Klisic J, Hu MC, Nief V, Reyes L, Fuster D, Moe OW, et al. Insulin activates $\mathrm{Na}+/ \mathrm{H}+$ exchanger 3: Biphasic response and glucocorticoid dependence. Am J Physiol Renal Physiol 2002;283:F532-9. 
[28] Asplin JR. Uric acid stones. Semin Nephrol 1996;16:412-24.

[29] Lemann Jr J, Lennon EJ, Piering WR, Prien Jr EL, Ricanati ES. Evidence that glucose ingestion inhibit net renal tubular reabsorption of calcium and magnesium in man. J Lab Clin Med 1970;75:578-85.

[30] Bonora E, Targher C, Alberiche M, Formentini G, Calcaterra F, Lombardi S, et al. Predictors of insulin sensitività in type 2 diabetes mellitus. Diabet Med 2002;19:535-42.
[31] Cohen O, Epstein GS, Weisz B, Homko CJ, Sivan E. Longitudinal assessment of insulin sensitivity in pregnancy. Validation of the homeostasis model assessment. Clin Endocrinol 2006;64:640-4.

[32] Becker B, Kronenberg F, Kielstein JT, Haller H, Morath C, Ritz E, et al. Renal insulin resistance syndrome, adiponectin and cardiovascular events in patients with kidney disease: the mild and moderate kidney disease study. J Am Soc Nephrol 2005;16:1091-8. 